\title{
Spatio-spectral Representation Learning for Electroencephalographic Gait-Pattern Classification
}

\author{
Sim Kuan Goh, Hussein A. Abbass, Senior Member, IEEE, Kay Chen Tan, Fellow, IEEE, Abdullah \\ Al-Mamun, Senior Member, IEEE, Nitish Thakor, Fellow, IEEE, Anastasios Bezerianos, Senior Member, IEEE, \\ and Junhua Li*, Senior Member, IEEE
}

\begin{abstract}
The brain plays a pivotal role in locomotion by coordinating muscles through interconnections that get established by the peripheral nervous system. To date, many attempts have been made to reveal the underlying mechanisms of humans' gait. However, decoding cortical processes associated with different walking conditions using EEG signals for gait-pattern classification is a less-explored research area. In this paper, we design an EEG-based experiment with four walking conditions (i.e., free walking, and exoskeleton-assisted walking at zero, low and high assistive forces by the use of a unilateral exoskeleton to right lower limb). We proposed spatio-spectral representation learning (SSRL), a deep neural network topology with shared weights to learn the spatial and spectral representations of multi-channel EEG signals during walking. Adoption of weight sharing reduces the number of free parameters, while learning spatial and spectral equivariant features. SSRL outperformed state-of-the-art methods in decoding gait patterns, achieving a classification accuracy of $\mathbf{7 7 . 8 \%}$. Moreover, the features extracted in the intermediate layer of SSRL were observed to be more discriminative than the hand-crafted features. When analyzing the weights of the proposed model, we found an intriguing spatial distribution that is consistent with the distribution found in well-known motor-activated cortical regions. Our results show that SSRL advances the ability to decode human locomotion and it could have important implications for exoskeleton design, rehabilitation processes and clinical diagnosis.
\end{abstract}

This work was supported by the Ministry of Education of Singapore under the grant MOE2014-T2-1-115, the Australian Research Council grant number DP160102037, and the Research Grants Council of the Hong Kong Special Administrative Region, China [Project No. CityU11202418]. The authors also thank the National University of Singapore for supporting the Cognitive Engineering Group at the Singapore Institute for Neurotechnology (SINAPSE) under the grant R-719-001-102-232.

Sim Kuan Goh is with the Singapore Institute for Neurotechnology (SINAPSE) and Department of Electrical and Computer Engineering, National University of Singapore, 28 Medical Drive, 117456, Singapore. (simkuan@u.nus.edu, simkuangoh.bci@gmail.com)

Hussein A. Abbass is with the Trusted Autonomy Group, University of New South Wales, School of Engineering and Information Technology, Canberra, ACT 2600, Australia.

Kay Chen Tan is with the Department of Computer Science, City University of Hong Kong, Kowloon Tong, Kowloon, Hong Kong.

Abdullah A. Mamun is with the Department of Electrical and Computer Engineering, National University of Singapore, 4 Engineering Drive, 117583, Singapore.

Nitish Thakor and Anastasios Bezerianos are with the Singapore Institute for Neurotechnology (SINAPSE), National University of Singapore, 28 Medical Drive, 117456, Singapore

Junhua $\mathrm{Li}$ is with the Singapore Institute for Neurotechnology (SINAPSE), National University of Singapore, 28 Medical Drive, 117456, Singapore, and is also with the Laboratory for Brain-bionic Intelligence and Computational Neuroscience, Wuyi University, Jiangmen, 529020, China, and the Centre for Multidisciplinary Convergence Computing (CMCC), School of Computer Science and Engineering, Northwestern Polytechnical University, Xi'an, 710072, China.

* indicates the corresponding author (juhalee@nus.edu.sg, juhalee.bcmi@gmail.com)
Index Terms-Spatio-spectral Representation Learning, Electroencephalogram (EEG), Exoskeleton, Gait Pattern, Convolutional Neural Network.

\section{INTRODUCTION}

The human locomotor system performs a variety of movements (e.g., walking, running) intuitively and remarkably on many terrain types. Nevertheless, we still have difficulties in elucidating the inner workings of achieved movements. Despite much research effort, the fundamental principles that govern the intricate underlying mechanism of human gait have yet to be discovered [1]. Revealing more locomotion principles has many practical implications for patients with gait impairment that include the design of rehabilitation-oriented devices [2], [3], gait correction treatment [4] and clinical diagnosis [5]. The understanding of neural mechanisms in a human gait is also indispensable to enabling an intuitive use of robotic exoskeleton for durable strength augmentation in the lower limb [3]. Electroencephalographic (EEG) signals have been used to study and monitor brain activities due to their portability and high temporal resolution. EEG signals are susceptible to different kinds of artifacts (e.g., eye blink and head movement artifacts), but a number of artifact removal techniques (e.g. regression-based [6], surrogate-based [7], adaptive filtering [8], widely used independent component analysis (ICA) [9] and the methods reviewed in [10]) have been developed to mitigate these inherent noises [11], [12].

Numerous EEG studies have revealed gait-related cortical activation, known as event-related (de)synchronization (ERD/ERS) [13], when subjects performed motor imagery of gait [14], actual ankle movements [15], walking on a treadmill [16] and lower limb movement on a dynamic tilt table [1]. Both imagined and real movements activated similar brain regions including primary motor cortex (M1), primary somatosensory cortex (S1) and supplemental motor area (SMA) [1]. The association between these regions and lower limb movements was also revealed using neuroimaging modalities such as functional magnetic resonance imaging (fMRI) [17], [18], [19] and functional near-infrared spectroscopy (fNIRS) [20].

In these gait-related brain regions, ERD and ERS in alpha and beta rhythms were found to be prominent and consistent across many gait studies [21], [22]. A walking balance study has demonstrated that beta oscillation decreased while theta oscillation increased in the sensorimotor cortex [16]. The modulation of gamma band in relation to the gait cycle was 
observed in the central sensorimotor area [23], [24]. These findings suggest that it is possible to associate and classify human gait patterns by extracting gait-related spatial and spectral features from EEG signals.

Classification of mental tasks and conditions [25] (e.g., motor imagery [26], P300, steady-state visually evoked potentials (SSVEP) [27]) based on EEG can be achieved by classical machine learning, by combining unsupervised learning and supervised learning. Unsupervised learning methods (e.g., ICA) act as feature extraction and dimensionality reduction techniques, which is an important preliminary step for predictive modelling using a supervised learning approach (e.g., support vector machine (SVM) [28] and random forest (RF) [29]).

Deep learning models simultaneously integrate and optimize feature extraction and predictive modelling using deep neural network. These models have enormous growth in popularity and practicality for complex tasks such as image captioning [30], generating images [31] and defeating human champions in games like "Go" game [32]. While deep learning has been successfully applied to image, text, speech and video data that have abundant data, a few pioneering studies have reported the effectiveness of deep learning in EEG analysis.

Stacked denoising autoencoders were used for EEG feature learning in motor imagery [33], [34], emotion recognition [35] and joint EEG-Electromyography (EMG) study [36]. Convolutional neural networks (CNN) [37] architectures were employed for P300 [38] and epileptic seizure prediction [39], [40]. Motor imagery task was studied [41] using restricted boltzmann machine [42], [43]. A recurrent neural network (RNN) [44] model was used for sleep stage prediction [45] and epileptic seizure detection [46]. Long short-term memory (LSTM), a special case of RNN, was used to model the dynamics of working memory [47] and detection of epileptic seizure [48], and sleep stage classification [49].

Research on decoding human gait pattern based on EEG, however, remains largely unexplored. Our previous work [50] used EEG and a lower limb exoskeleton [51] to study brain activities related to human gait. In the experiment, wet electrodes and wired EEG system were used to record signals. This setting ensured high signal quality, but reduced the mobility. A trolley had to be employed to carry the equipment. Alternatively, emerging dry wireless EEG system can be used in the walking experiment or operational environments [52]. It is suitable to record signals in a mobile manner such as brain assessment while moving in a supermarket [53]. However, the signal-to-noise ratio is relatively lower compared to the wet wired EEG system. A classical classier, SVM, was employed to recognize gait patterns based on EEG for three types of walking. In this paper, we extend the study in [50] by developing an end-to-end deep learning model for classifying four walking conditions (i.e., free walking and exoskeleton-assisted walking at zero, low and high assistive forces). Twenty EEG channels that cover sensorimotor region [1] were analyzed. As gait-related brain activities exhibit spatial [1] and spectral [21], [22] characteristics, we propose spatio-spectral representation learning (SSRL), a deep neural network topology with shared weights, to capture these intrinsic features while performing predictive modelling simultaneously. Adopting the weight sharing mechanism in convolutional neural network to handle positional changes of objects in images and reduce the number of free parameters (weights), SSRL learns a series of spatial and spectral filters with shared weights to capture discriminative spectral features across spatial locations by supervised learning. The performance of our proposed model was evaluated and compared to state-of-the-art machine learning techniques. Moreover, a further appraisal was performed to evaluate the discriminative power of learned features in the intermediate layers of SSRL. Furthermore, we explore the learnable weights in the SSRL to demonstrate whether the model finds the brain regions classically associated with human gait.

The rest of this paper is organized as follows: Section II presents the experiment, data preprocessing, and SSRL. In Section III, SSRL is evaluated and compared to state-of-theart machine learning techniques. Results and discussion are presented in Sections IV and V, respectively. Conclusions are drawn in Section VI.

\section{Methodology}

\section{A. Experimental Protocol}

In the experiment, subjects performed free walking (FW, normal walking without exoskeleton support) and exoskeletonassisted walking at zero force ( $\mathrm{ZF}$, walking with an exoskeleton, but no torque assistance is provided), low assistive force (AFL, walking with an exoskeleton that provides the low assistive torque) and high assistive force (AFH, walking with an exoskeleton that provides the high assistive torque) along an approximately 70 feet corridor. The exoskeleton [51] was attached on their right lower limb. EEG signals were recorded by an ANT ASA-Lab system (ANT BV, Netherlands). Sixtytwo wet electrodes were used and connected to the ANT amplifier by a cable.

This study recruited 30 healthy male subjects from National University of Singapore (NUS). Due to incompleteness in recording, the data from 3 subjects were excluded. The average age of all subjects was $24 \pm 2.32$ and their average body mass index (BMI) was 22.92 \pm 2.76 . All of them had normal vision or corrected-to-normal vision with no history of lower limb injury. The consent form was signed after receiving thorough explanations of the procedure. The experimental protocol was reviewed and approved by Institutional Review Board of NUS.

\section{B. EEG Preprocessing}

EEG signals were recorded at a sampling rate of $1000 \mathrm{~Hz}$ and referenced to the right earlobe. The signals were centered to zero-mean for each channel and down-sampled to $250 \mathrm{~Hz}$. A band-pass filter with cutoff frequencies of $0.5-45 \mathrm{~Hz}$ was then applied. The least mean squares [8] was used to reduce the influence of ocular artifact and the canonical correlation analysis-based method [54] was utilized to mitigate EMG effect on EEG. After rejecting heavily artifact-contaminated segments, ICA was employed to remove the independent components representing artifacts (e.g., EMG and other types 
of artifacts reported in [11]) by visual inspection. The average number of remaining ICs was $45.5 \pm 6.9$ (mean \pm standard deviation). Corrected EEG signals were reconstructed using the remaining independent components. EEG epochs of each walking condition were then extracted according to the EMG gait markers (the details of the procedure were reported in [55]). Abnormal gait cycles and short gait cycles (i.e., less than 1 second) were removed. The average number of the remaining gait cycles were $48.1 \pm 19.8$ (mean \pm standard deviation) for $\mathrm{ZF}, 46.3 \pm 15.9$ for $\mathrm{FW}, 45.7 \pm 15.9$ for $\mathrm{AFL}$ and $46.6 \pm 17.8$ for AFH.

250-point discrete Fourier transform (FT) was performed on the first and last 250 points of an EEG gait segment for all channels, followed by an averaging of the two magnitude spectra. The montage of the inclusive channels was shown in Fig. 1. Two types of spectral ranges were studied, wide spectral (WS, 1-42Hz) range and prominent spectral (PS, $8-30 \mathrm{~Hz})$ range. The latter covered mu and beta bands that were prominent in motor-related EEG studies [21], [22]. Data (channel $\times$ spectral width) D-WS of size $20 \times 42$ and D-PS of size $20 \times 42$ were obtained. In addition, EEG spectrum can also be divided into delta $(1-4 \mathrm{~Hz})$, theta $(4-7 \mathrm{~Hz}), \mathrm{mu}(8-12 \mathrm{~Hz})$, beta $(13-30 \mathrm{~Hz})$ and gamma $(30-42 \mathrm{~Hz})$ bands to obtain compact data matrix D-WS-C of size $20 \times 5$ and D-PS-C of size $20 \times 2$. This step is also widely known as feature engineering and hand-craft feature design where expert knowledge is required. Eventually, data is normalized to zero mean and unit variance based on the values calculated from the training data in a 5fold cross-validation setting.

\section{Spatio-spectral Representation Learning}

Weight sharing mechanism is essential in convolutional neural network to learn local features in images where objects of interest might appear at different position in different images. Moreover, it adds a parsimonious pressure to allow the network to generalize better with controlled-complexity [56]. In this paper, weight sharing mechanism is adapted for spatiospectral EEG data to extract spatial features across frequency and spectral features across spatial locations. The architecture of the SSRL is illustrated in Fig. 1. It is a neural network with 3 hidden layers that comprises two weight sharing (spatial and spectral) layers and fully connected layers. Softmax activation function is used in the output layer. In addition, leaky rectified linear unit (ReLU) activation function and dropout are used in each hidden layer.

Each spatial or spectral filter $f$ in shared weight layers $l$ performs convolution on the output of the previous layer $y^{l-1}$ with bias terms, non-linear activation functions and dropout to compute $y^{l}$. The convolution can be expressed as follows:

$$
x_{i, j, f}^{l}=\sum_{a=0}^{n-1} \sum_{b=0}^{m-1} w_{a, b, f}^{l} \times y_{i+a, j+b, f}^{l-1}+b_{f}^{l}
$$

where the filter $f$ of size $n \times m$ is applied across sub-regions of $y^{l-1}$ of size $N \times M$ and produces output $x^{l}$ of size $(N-n+$ $1) \times(M-m+1)$. Fig. 1 illustrates the convolution operations in the first two layers performed on D-WS or D-PS of size $C \times$ $F$. The size of a spatial filter equals to number of electrodes $C$,
TABLE I

SSRL PARAMETERS.

\begin{tabular}{ll}
\hline Parameters & Settings \\
\hline No. of Spatial Filters $M_{1}$ & 10 \\
No. of Spectral Filters $M_{2}$ & 10 \\
Size of FC Layers & 20,4 \\
Adam Learning Rate & $10^{-3}$ \\
Dropout Iteration & $90 \%$ or $100 \%$ \\
Dropout Probability & $0 \%$ to $95 \%$ \\
Leaky ReLU Coefficient & 0.2 \\
\hline
\end{tabular}

while the size of a spectral filter equals to number of frequency bins $F$. Non-linear activation functions (e.g., ReLU) can then be applied on $x^{l}$. ReLU is defined as follows:

$$
\sigma(x)=x^{+}=\max (0, x)
$$

However, ReLU suffers from the phenomenon of "dying ReLU," where neurons will never activate during training. Leaky ReLU [57], which is an attempt to fix this phenomenon, uses a small negative slope $l$ for $x<0$, as follows:

$$
\sigma(x)=\left\{\begin{array}{cc}
x, & \text { if } x>0 \\
l x, & \text { otherwise }
\end{array}\right.
$$

For $l<1$, it is equivalent to:

$$
\sigma(x)=\max (x, l x)
$$

The output of the convolutional layer $y^{l}$ can be computed as follows:

$$
y_{i, j, f}^{l}=m_{i, j, f}^{l} \times \sigma\left(x_{i, j, f}^{l}\right)
$$

where $m_{i, j, f}^{l}$ is a binary dropout [58] mask with dropout probability $D_{p}$ in the training phase and 0 in the testing phase. $m_{i, j, f}^{l}$ is a scaled mask matrix of independent Bernoulli variable:

$$
m_{i, j, f}^{l} \sim \frac{\text { Bernoulli }\left(1-D_{p}\right)}{1-D_{p}}
$$

Softmax activation function was used to turn the output $z$ from neural network into predicted class probabilities:

$$
f(z)_{i}=\frac{e^{z_{i}}}{\sum_{k} e^{z_{k}}}
$$

The training of SSRL can then be achieved by minimizing the cost function $C=\sum_{i} L_{i}$, where $L_{i}$ is the cross-entropy loss of class $i$ :

$$
L_{i}=-\log \left(f(z)_{i}\right)
$$

Optimizing cross-entropy is the same as optimizing Kullback-Leibler divergence [59]; which makes the predicted output distribution close to the true distribution of the training data. Adam algorithm [60], an efficient stochastic optimization technique, was utilized to optimize the cost function.

In addition to dropout probability $D_{p}$ that determines the probability of a neuron being dropped out from the network, 


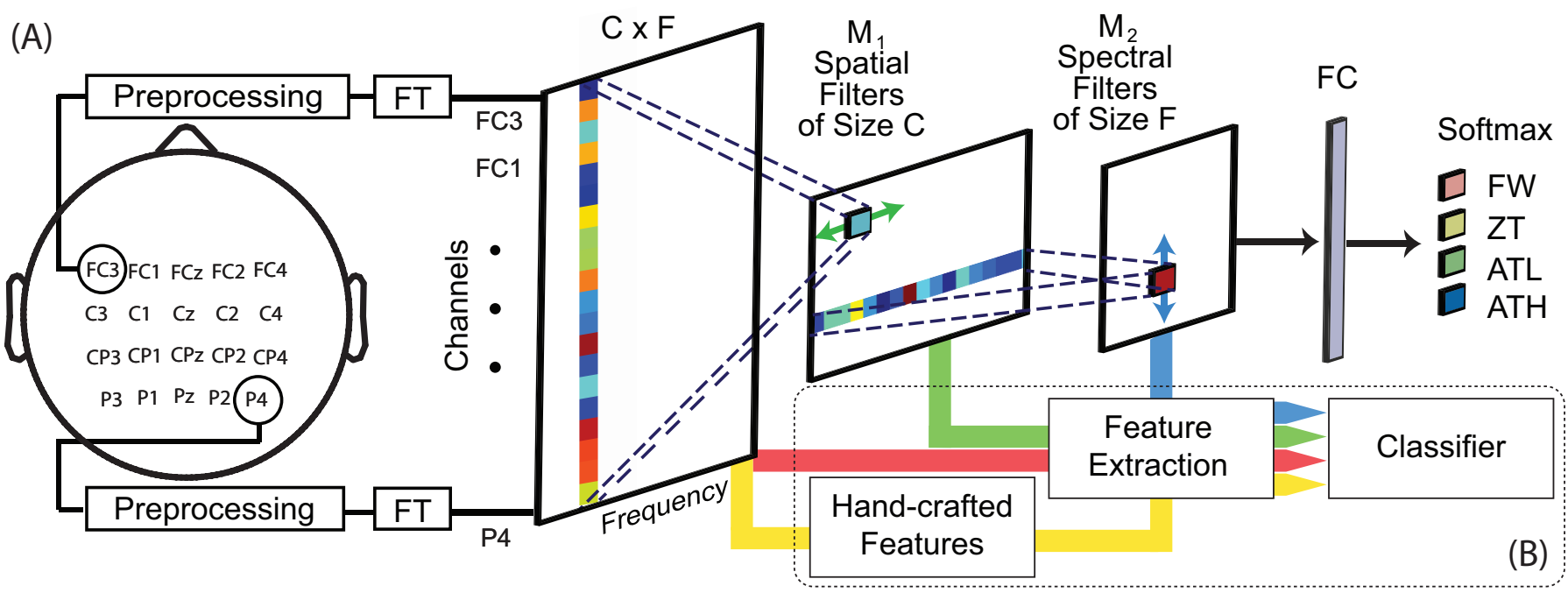

Fig. 1. The architecture of the spatio-spectral representation learning (SSRL). The SSRL was trained to classify 4 different walking conditions using frequency domain features of multi-channel EEG signal (Channels $\times$ Frequency). It comprises two weight sharing layers with spatial and spectral filters, fully connected layers (FC) and output layer with softmax activation functions. In addition, leaky ReLU activation functions and dropout are employed in each hidden layer. (B) The illustration of classical machine learning method for classification. The method was utilized to obtain baseline performance, as well as a tool for evaluating the discriminative power of features extracted by the SSRL.

we investigated dropout iteration $D_{i}$ that determines the percentage of training iterations where dropout is applied to the network. For instance, dropout is used 9 out of 10 training iterations for $D_{i}$ of $90 \%$.

Fully connected layer connects every neuron in one layer to every neuron in its previous layer. The proposed model can be summarized by writing all operations that map input data to prediction into a sequence: Data D-WS or D-PS $\rightarrow$ SpatialFilter $\rightarrow$ Leaky ReLU $\rightarrow$ Dropout $\rightarrow$ Spectral-Filter $\rightarrow$ Leaky ReLU $\rightarrow$ Dropout $\rightarrow$ FC $\rightarrow$ Leaky ReLU $\rightarrow$ Dropout $\rightarrow$ FC $\rightarrow$ Softmax $\rightarrow$ Predicted Classes

Table I summarizes the parameters of SSRL. Cost function smaller than $10^{-10}$ and training iterations of $10^{4}$ were used as the stopping criteria.

\section{Evaluation}

To evaluate the performance of SSRL on D-WS and D-PS, we compare it against classical machine learning techniques (i.e., RF and SVM) that exploit hand-crafted and unsupervised features for classification. The hyperparameters of these techniques, shown in Table II, were fine-tuned using 5-fold cross-validation and grid-search.

\section{A. Feature Selection and Extraction}

Dimensionality reduction techniques improve the performance of classifiers, as they reduce the number of features to mitigate against curse of dimensionality. These techniques can be categorized into feature selection (e.g., F-score) that selects subset of original features and feature extraction (e.g., principal component analysis (PCA)) that derives new features from original features. The first category does not change the nature of the features, while in the second category, mathematical transformations are used to reduc e the number of features.
In this study, F-score, was used for the hand-crafted features D-PS-C and D-WS-C while PCA, was applied to D-PS and D-WS. F-score ranks features based on a weighted average of precision and recall, whereas PCA linearly transforms data to a set of orthogonal basis that captures the directions of variations in the data. Dimensionality reduction was achieved using F-score by selecting a subset of the top discriminative features and PCA by reconstructing eigenvectors that have the highest eigenvalues.

\section{B. Random Forest}

A random forest (RF) is an ensemble of random decision trees built to perform classification and regression tasks. Combining bootstrap sampling of dataset and ensemble learners, RF has a higher generalization ability compared to decision tree. RF has been successfully applied to a diverse range of applications. In EEG, RF has been used to the classification of motor imagery [61], [62], [63], removal of muscle artifacts [64], an infant EEG monitoring system [65], detection of P300 [66], and early seizure [67]. As RF has been reported to have achieved competitive classification performance in these EEG studies, it was used as a baseline in this study.

\section{Support Vector Machine}

SVM is an optimal linear discriminative classier that provides maximum-margin hyperplanes defined by support vectors. Applying an appropriate kernel (such as radial basis function (RBF) and polynomial and hyperbolic tangent kernels) to SVM allows the algorithm to handle non-linear datasets. High classification performance has been reported using SVM or its variants in the studies of P300 [68], motor imagery [69], and seizure detection [70]. Due to its high efficiency, SVM with an RBF kernel has been widely used as a baseline to evaluate the generalization performance of other machine 
TABLE II

GRID SEARCH OF HYPERPARAMETER SETTINGS FOR SVM AND RF.

\begin{tabular}{lll}
\hline Parameters & Range & Step \\
\hline $\begin{array}{l}\text { No. of F-score features } \\
\begin{array}{l}\text { Percentage of kept PCA } \\
\text { components }\end{array}\end{array}$ & \# of features to 20 & -5 \\
RF & $10 \%$ to $100 \%$ & $10 \%$ \\
$\begin{array}{l}\text { No. of RF estimators } \\
\text { No. of RF features for } \\
\text { splitting }\end{array}$ & \# of features to 20 & -5 \\
SVM of features to 20 & -5 \\
SVM-C & \\
SVM- $\gamma$ & $2^{-5}$ to $2^{17}$ & \\
\hline
\end{tabular}

learning techniques. In the current study, SVM with RBF kernel were also used as a baseline.

\section{Implementation Details}

In our study, we have compared the classification performance between the SSRL and the classical machine learning methods, as well as, we have evaluated the discriminative power of features extracted at intermediate layers of the SSRL. Fig. 1 illustrated the evaluation scheme. A standard pipeline of feature extraction and classification was used when classical machine learning methods served as baseline methods. In the case of feature evaluation, the features extracted at intermediate layers were fed into the standard pipeline to obtain classification accuracies.

\section{Results}

The average testing accuracy of SSRL using a different dropout probability $D_{p}$ and dropout iteration $D_{i}$ was shown in Fig. 3. As $D_{p}$ increases, the accuracy curves showed an "inverted U-shape", with an initial rise, a peak at moderate $D_{p}$, and a decline. A slight decrease of dropout iteration $D_{i}$ from $100 \%$ to $90 \%$, which allowed SSRL to learn without dropout for $10 \%$ of the training iteration, slightly improved the overall performance. The settings that attained the highest accuracy were $D_{p}$ of $35 \%$ and $D_{i}$ of $90 \%$ for wide spectral D-WS and $D_{p}$ of $40 \%$ and $D_{i}$ of $90 \%$ for prominent spectral D-PS.

The classification accuracy of all methods, in their best parameter settings, are shown in Fig. 4 for all subjects. The average classification accuracy (mean \pm standard error) of four methods for prominent spectral range D-PS were $58.9 \pm 1.7 \%$ for RF-FS, $54.8 \pm 2.0 \%$ for SVM-PCA, $70.2 \pm 1.6 \%$ for SVMFS and $72.9 \pm 1.7 \%$ for SSRL. Generally, there were increases in average classification accuracy when wide spectral range D-WS were used. RF-FS achieved $65.9 \pm 1.8 \%$; SVM-PCA achieved $64.3 \pm 1.8 \%$; SVM-FS achieved $74.3 \pm 1.6 \%$; SSRL achieved $77.8 \pm 1.8 \%$.

According to two-way analysis of variance (ANOVA), there were a significant spectral range effect $\left(F_{(1,208)}=26.2\right.$, $\left.p<1.0 \times 10^{-6}\right)$ and a classifier effect $\left(F_{(3,208)}=37.5\right.$, $\left.p<1.0 \times 10^{-18}\right)$ on classification accuracy. There was no significant interaction effect on classification accuracy between the spectral range factor and the classifier factor. The post-hoc two-tailed paired t-test, shown on the right panel of Fig. 4, revealed that the proposed approach significantly outperformed other methods in both cases of wide and prominent spectral range. SVM-FS and RF-FS were found to have accuracy higher than SVM-PCA. Fig. 2 summarized the classification accuracies of machine learning techniques using features extracted at intermediate layers of the SSRL. RF-FS achieved $70.5 \pm 1.0 \%$ in the first layer and $75.9 \pm 1.0 \%$ in the second layer; SVM-PCA achieved $69.0 \pm 0.9 \%$ in the first layer and $76.2 \pm 1.0 \%$ in the second layer; SVM-FS achieved $68.1 \pm 0.9 \%$ in the first layer and $76.3 \pm 1.0 \%$ in the second layer; Features in the second layer of SSRL were found to be more discriminative than the features in the first layer and hand-crafted features.

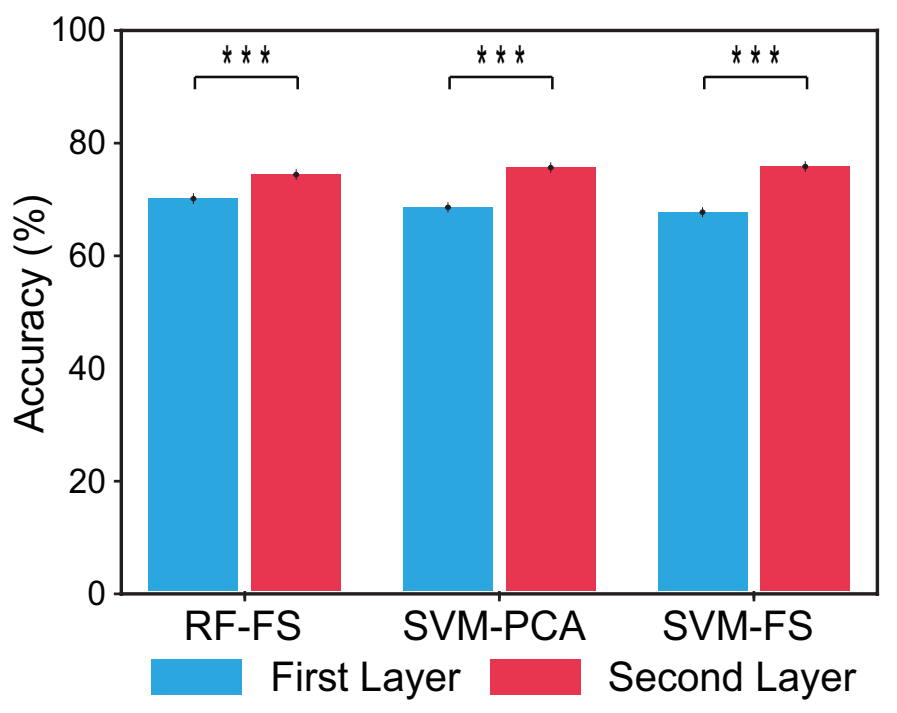

Fig. 2. Discriminative power evaluation of features extracted at intermediate layers of the SSRL in terms of classification accuracies derived from RF-FS, SVM-PCA, and SVM-FS $\left(* * *\right.$ indicates $\left.p<10^{-6}\right)$.

To study and interpret the topographic features learned by the filters in the SSRL, we analyzed the learnable weights in the first hidden layer that captured spatial information. Individual spatial filter, average spatial filter across 5-fold cross-validation for a subject and the grand average spatial filter across all subjects at EEG channel $i$ can be obtained as follows:

$$
G P_{S, F, P}(i)=\frac{1}{N} \sum_{S} \sum_{F} \sum_{P} \sum_{j}\left|w_{S, F, P}(i, j)\right|
$$

where each weight $w_{S, F, P}(i, j)$ was normalized between zero and one using Min-Max scaling. $N$ denotes the total number of filters, $S$ denotes number of subjects, $F$ denotes 5 -fold cross-validation and $P$ denotes number of spatial filters for a specific subject and fold. Fig. 5 and supplementary Fig. 1 visualized the grand average spatial filter across all subjects (27 subjects), a few instances of the spatial filters across 5-fold cross-validation of individual subjects and individual spatial filters of a subject for both cases of wide spectral D-WS 
and prominent spectral D-PS range. The means and standard deviations of spatial filters for each channel were listed in supplementary Table I.

Other subjects were observed to have similar spatial filters, shown in supplementary Fig. 2 and 3. Activation around the left sensorimotor region was observed for the majority of subjects. Moreover, the average spatial filters between D-PS and D-WS were observed to be similar.

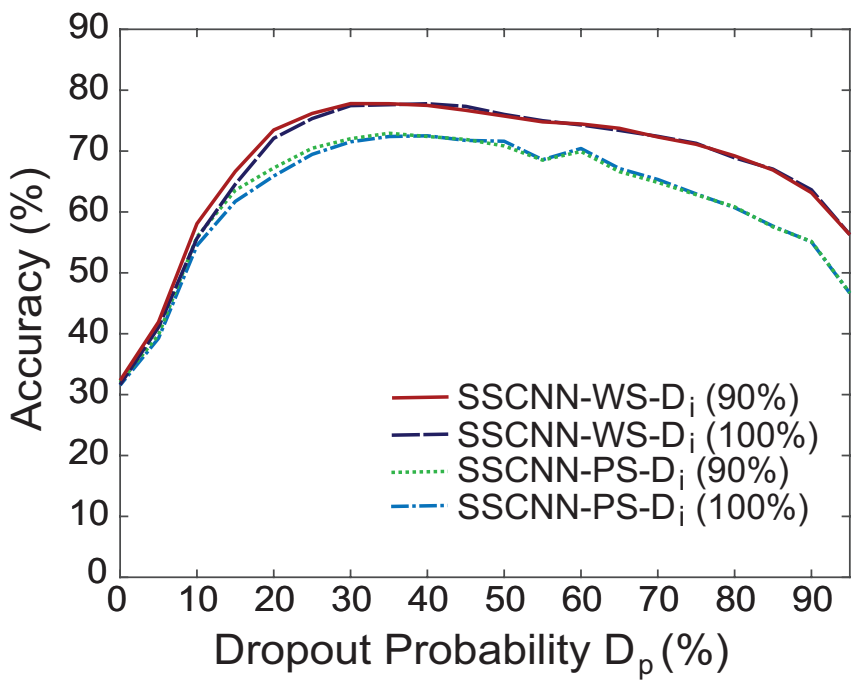

Fig. 3. 5-fold cross-validation accuracy (\%) curves of SSRL using different dropout probability $D_{p}$ and dropout iteration $D_{i}$ in percentage for both cases of wide spectral (WS, 1-42Hz) range and prominent spectral $(\mathrm{PF}, 8-30 \mathrm{~Hz})$ range.

\section{DISCUSSION}

Comparing the classification accuracy of the methods described in Section III, the overall testing accuracy of SVMFS and RF-FS were higher than SVM-PCA in both cases of wide spectral and prominent spectral ranges. This difference is because SVM-PCA used unsupervised learning PCA for dimensionality reduction while SVM-FS and RF-FS utilized prior EEG knowledge (i.e., EEG spectrum can be divided into typical bands including delta, theta, alpha, beta and gamma) and F-score for classification. Thus, the results of the comparison suggested that hand-crafted feature design played an important role in the performance of SVM and RF. On the contrary, the topology of SSRL was able to learn the spatial and spectral invariant features directly from the input without the burden of hand crafted features and dimensionality reduction, achieving the highest classification accuracy in both cases of prominent and wide spectral range. The classification performance of machine learning pipelines using features extracted at intermediate layers of the SSRL further confirms that the proposed method extracted feature at least as good as hand-crafted features, even the number of features in the second layer is the same as the number of hand-crafted features.

While the prominent spectral range has been consistently reported to be associated with lower limb movements [21], [22], limited literature reported the modulation of other bands (e.g., delta [71], theta [21], gamma [23]) in human gait. Making an attempt to investigate this, we compared the classification models that were trained on both cases of prominent frequency range and wide spectral range. It was found that the incorporation of EEG bands (i.e., delta, theta, gamma) to the prominent spectral range resulted in an increase in accuracy for all four methods, and hence suggested that these additional bands also carries gait pattern information for additional discriminative power.

Beyond gait pattern recognition, another advantage of the SSRL lies in its competency to learn discriminative EEG representations. As each spatial filter in SSRL can be considered as a spatial feature extractor that influences gait pattern classification, semantic interpretation of the weights can provide discriminative spatial information and possibly locate brain regions responsible to gait control of different walking conditions. A few instances of individual spatial filters were shown in panel (C) of Fig. 5 and supplementary Fig. 1.

These spatial filters were observed to have highlighted diverse brain regions. While each of these filters learned diverse spatial features, the main brain region associated with walking tasks can be obtained by averaging the individual spatial filters. The average individual spatial filter for a subject, shown in panel (B) of Fig. 5 and supplementary Fig. 1, highlighted brain activities around the left sensorimotor region and suggested that this region had more discriminative power for clas sifying gait patterns. We also explored the grand average spatial filter across all subjects and found contralateral brain activation in the sensorimotor cortex on the left hemisphere that corresponds to the right lower limb with the exoskeleton. This region is in agreement with the findings of previous studies [1], [72]. These findings could prove useful for designing an EEGbased gait system as a customized electrode placement with higher spatial resolution in this gait-related brain region.

Although the SSRL showed superior classification performance compared to fine-tuned SVM and RF, it also faced the same limitation of deep neural networks when it comes to parameter tuning. The computational cost for deep neural network grows exponentially as the number of parameters increases. A prevalent manner of determining deep learning architecture and corresponding parameters is based on experience. However, deep neural network, with an adequate representational capacity (e.g., network depth), regularization (e.g., dropout, $L_{1}$ and $L_{2}$ regularization) and training, can usually achieve a good performance as reported in many applications. Based on our experience, the performance does not change dramatically given that parameters are not set to extreme values. In this study, moderate depth and dropout allowed SSRL to exceed the classification accuracy of classical machine learning techniques. In addtion, weight sharing mechanism of SSRL reduces the number of free parameters and computational complexity. For instance, conventional neural network with no hidden layer (i.e. fully connected network and SVM) has 3364 parameters for tuning and the number of parameters is increased with the increase of the number of hidden layers. However, SSRL with three hidden layers only has 2744 parameters.

In summary, the results demonstrated several benefits of 

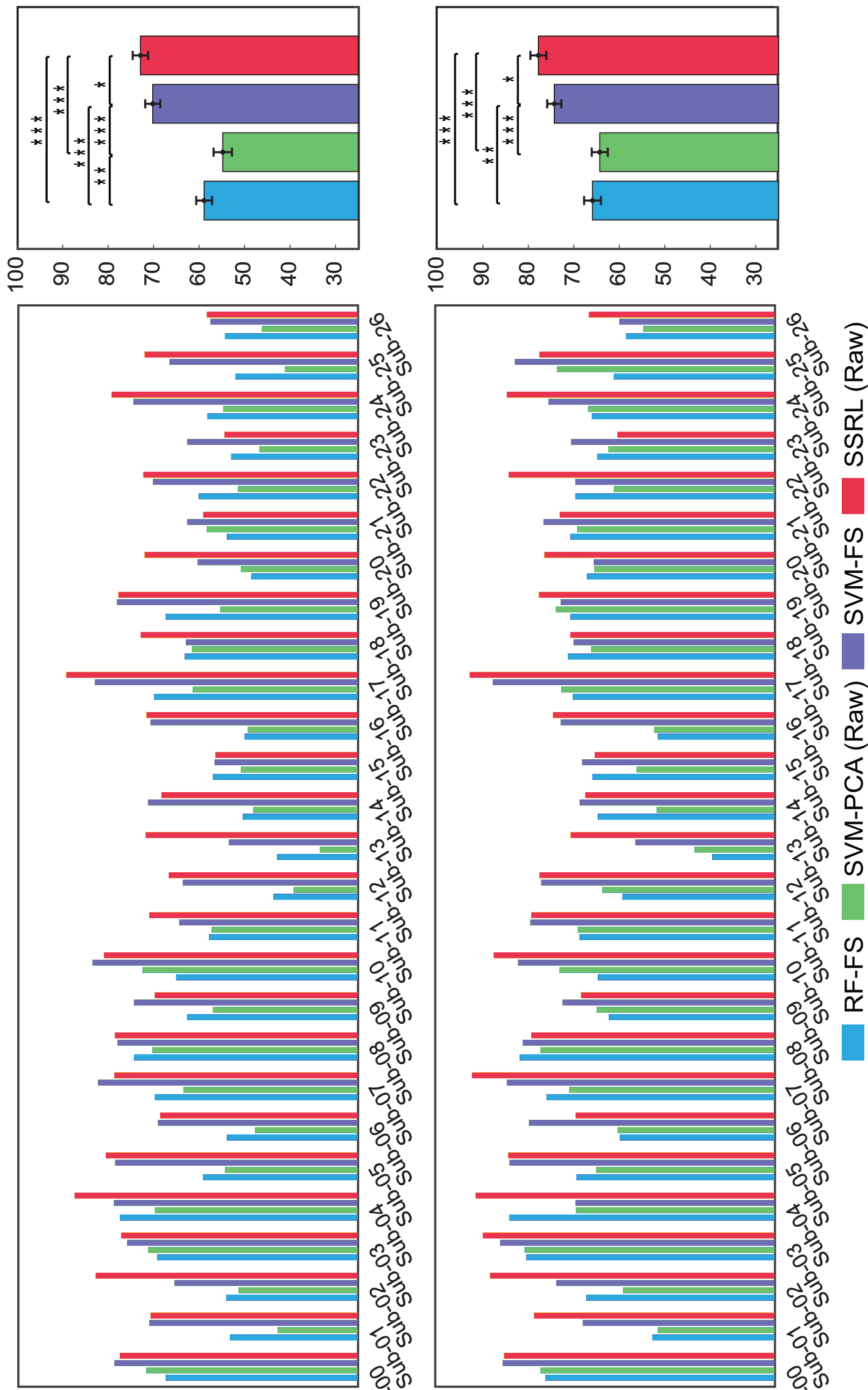

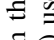

ธิธี

हं 

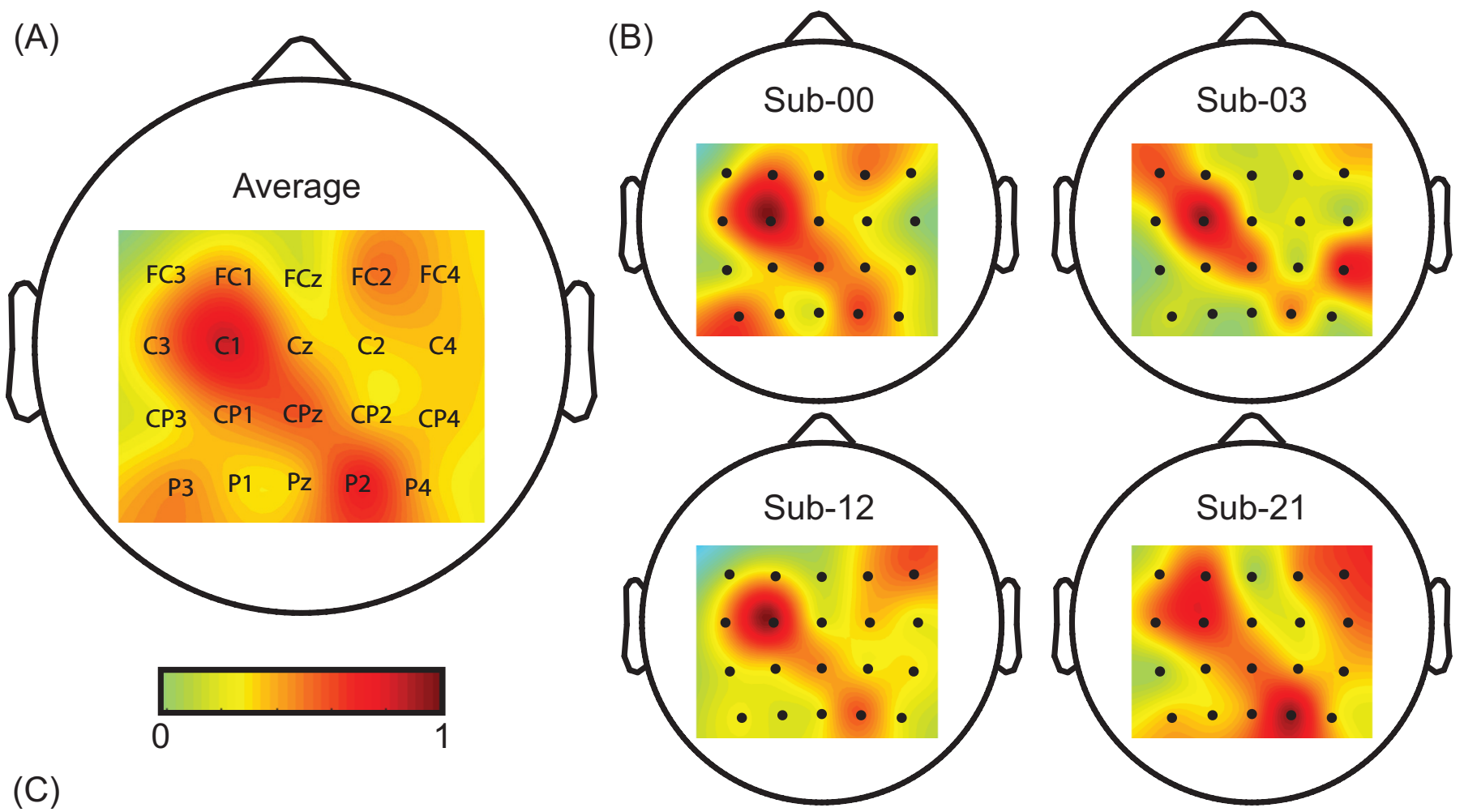

(C)
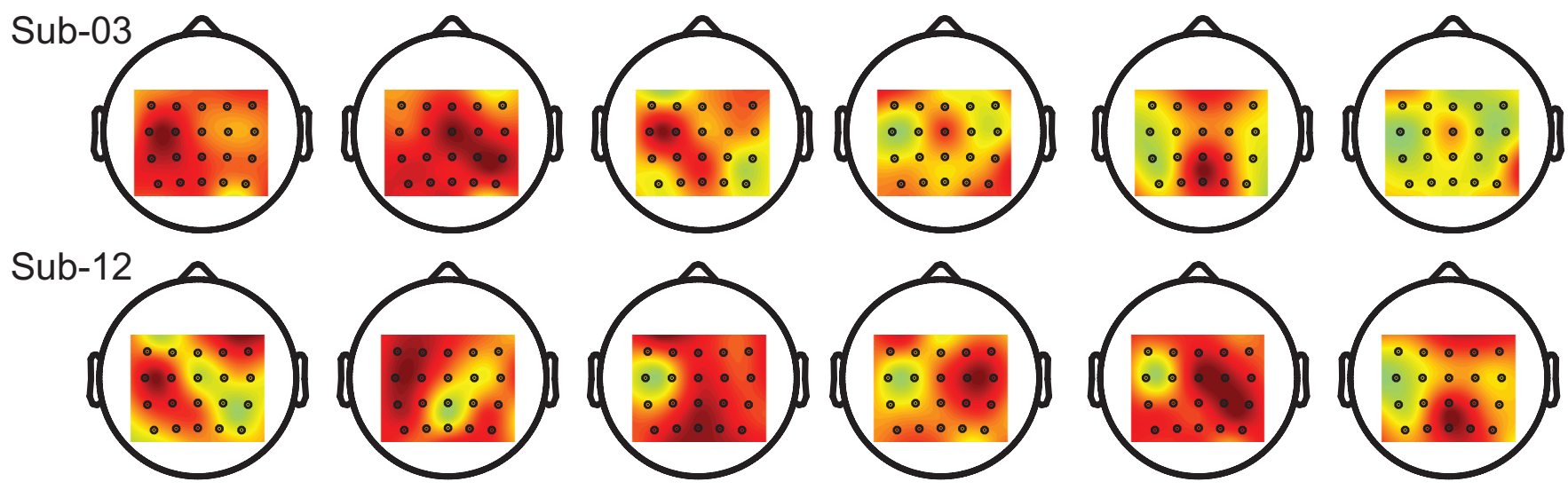

Fig. 5. Topographies of spatial filters learnt in SSRL on D-WS with $D_{p}$ of $35 \%$ and $D_{i}$ of $90 \%$. (A) Grand average spatial filter across all subjects (27 subjects). (B) Average spatial spatials of individual subjects. (C) Spatial filters from two representative subjects (subject 3 and subject 12).

SSRL compared to classical machine learning techniques that include (1) higher classification accuracy; (2) ability to learn comprehensible topographic features that are associated with brain involvement in gait control; (3) no burden of handcrafted feature engineering.

\section{CONCLUSION}

In this work, we proposed SSRL for human gait recognition that automatically learns spatial and spectral information contained in EEG signals. The spatial and spectral filters of SSRL utilize the weight sharing mechanism in convolutional neural network. More specifically, instead of learning filters for handling positional changes of objects in images, SSRL learns discriminative spectral features across spatial locations from multi-channel ERD/ERS. The proposed approach outperformed state-of-the-art machine learning techniques, achieving a classification accuracy of $77.8 \%$. In addition, SSRL discovered and highlighted well-known gait-related brain regions that were responsible for the physical coordination of walking with(out) the support of exoskeleton attached to the right lower limb. Our results demonstrated the advantages of SSRL in EEG-based gait recognition and multi-channel EEG representation learning.

As a future direction, the topology of SSRL can be further improved for cross-subject gait pattern classification. Moreover, it will be a challenging task to develop a new topology for deep neural network that can preserve the geometry of EEG electrode placement in a 3-dimensional space. The aforementioned progressions could enhance the diagnosis of abnormal gait and help administer appropriate rehabilitation process for restoring lower limb functions. 


\section{REFERENCES}

[1] M. Wieser, J. Haefeli, L. Bütler, L. Jäncke, R. Riener, and S. Koeneke, "Temporal and spatial patterns of cortical activation during assisted lower limb movement," Experimental brain research, vol. 203, no. 1 pp. 181-191, 2010.

[2] G. Aguirre-Ollinger, J. E. Colgate, M. A. Peshkin, and A. Goswami, "Design of an active one-degree-of-freedom lower-limb exoskeleton with inertia compensation," The International Journal of Robotics Research, vol. 30, no. 4, pp. 486-499, 2011.

[3] R. Jimenez-Fabian and O. Verlinden, "Review of control algorithms for robotic ankle systems in lower-limb orthoses, prostheses, and exoskeletons," Medical engineering \& physics, vol. 34, no. 4, pp. 397-408, 2012.

[4] S. K. Banala, S. H. Kim, S. K. Agrawal, and J. P. Scholz, "Robot assisted gait training with active leg exoskeleton (alex)," IEEE Transactions on Neural Systems and Rehabilitation Engineering, vol. 17, no. 1, pp. 2-8, Feb 2009.

[5] J. Verghese, C. Wang, R. B. Lipton, R. Holtzer, and X. Xue, "Quantitative gait dysfunction and risk of cognitive decline and dementia," Journal of Neurology, Neurosurgery \& Psychiatry, vol. 78, no. 9, pp. 929-935, 2007.

[6] G. Di Flumeri, P. Aricó, G. Borghini, A. Colosimo, and F. Babiloni, "A new regression-based method for the eye blinks artifacts correction in the eeg signal, without using any eog channel," in Engineering in Medicine and Biology Society (EMBC), 2016 IEEE 38th Annual International Conference of the. IEEE, 2016, pp. 3187-3190.

[7] M. Chavez, F. Grosselin, A. Bussalb, F. D. V. Fallani, and X. NavarroSune, "Surrogate-based artifact removal from single-channel eeg," IEEE Transactions on Neural Systems and Rehabilitation Engineering, vol. 26, no. 3, pp. 540-550, 2018.

[8] P. He, G. Wilson, and C. Russell, "Removal of ocular artifacts from electro-encephalogram by adaptive filtering," Medical and biological engineering and computing, vol. 42, no. 3, pp. 407-412, 2004.

[9] T.-W. Lee, M. Girolami, and T. J. Sejnowski, "Independent component analysis using an extended infomax algorithm for mixed subgaussian and supergaussian sources," Neural computation, vol. 11, no. 2, pp. 417-441, 1999.

[10] J. A. Urigüen and B. Garcia-Zapirain, "Eeg artifact removalstate-of-theart and guidelines," Journal of neural engineering, vol. 12, no. 3, p. 031001, 2015.

[11] S. K. Goh, H. A. Abbass, K. C. Tan, A. Al-Mamun, C. Wang, and C. Guan, "Automatic eeg artifact removal techniques by detecting influential independent components," IEEE Transactions on Emerging Topics in Computational Intelligence, vol. 1, no. 4, pp. 270-279, Aug 2017.

[12] S. K. Goh, H. A. Abbass, K. C. Tan, A. Al-Mamun, C. Guan, and C. C. Wang, "Multiway analysis of eeg artifacts based on block term decomposition," in Neural Networks (IJCNN), 2016 International Joint Conference on. IEEE, 2016, pp. 913-920.

[13] G. Pfurtscheller and F. L. Da Silva, "Event-related eeg/meg synchronization and desynchronization: basic principles," Clinical neurophysiology, vol. 110, no. 11, pp. 1842-1857, 1999.

[14] K. Sacco, F. Cauda, L. Cerliani, D. Mate, S. Duca, and G. Geminiani, "Motor imagery of walking following training in locomotor attention. the effect of the tango lesson," NeuroImage, vol. 32, no. 3, pp. $1441-$ 1449,2006

[15] O. Ciccarelli, A. T. Toosy, J. F. Marsden, C. M. Wheeler-Kingshott, C. Sahyoun, P. M. Matthews, D. H. Miller, and A. J. Thompson, "Identifying brain regions for integrative sensorimotor processing with ankle movements," Experimental Brain Research, vol. 166, no. 1, pp. 31-42, Sep 2005.

[16] A. R. Sipp, J. T. Gwin, S. Makeig, and D. P. Ferris, "Loss of balance during balance beam walking elicits a multifocal theta band electrocortical response," Journal of Neurophysiology, vol. 110, no. 9, pp. 2050-2060, 2013.

[17] B. H. Dobkin, A. Firestine, M. West, K. Saremi, and R. Woods, "Ankle dorsiflexion as an fmri paradigm to assay motor control for walking during rehabilitation," Neuroimage, vol. 23, no. 1, pp. 370-381, 2004.

[18] C. Calautti and J.-C. Baron, "Functional neuroimaging studies of motor recovery after stroke in adults," Stroke, vol. 34, no. 6, pp. 1553-1566, 2003.

[19] M. Gandolla, N. S. Ward, F. Molteni, E. Guanziroli, G. Ferrigno, and A. Pedrocchi, "The neural correlates of long-term carryover following functional electrical stimulation for stroke," Neural plasticity, vol. 2016, 2016.
[20] I. Miyai, H. C. Tanabe, I. Sase, H. Eda, I. Oda, I. Konishi, Y. Tsunazawa, T. Suzuki, T. Yanagida, and K. Kubota, "Cortical mapping of gait in humans: a near-infrared spectroscopic topography study," Neuroimage, vol. 14, no. 5, pp. 1186-1192, 2001.

[21] J. Wagner, T. Solis-Escalante, P. Grieshofer, C. Neuper, G. Mller-Putz, and R. Scherer, "Level of participation in robotic-assisted treadmill walking modulates midline sensorimotor eeg rhythms in able-bodied subjects," NeuroImage, vol. 63, no. 3, pp. 1203 - 1211, 2012.

[22] G. R. Müller-Putz, D. Zimmermann, B. Graimann, K. Nestinger, G. Korisek, and G. Pfurtscheller, "Event-related beta eeg-changes during passive and attempted foot movements in paraplegic patients," Brain research, vol. 1137, pp. 84-91, 2007.

[23] M. Seeber, R. Scherer, J. Wagner, T. Solis-Escalante, and G. R. MüllerPutz, "Eeg beta suppression and low gamma modulation are different elements of human upright walking," Frontiers in human neuroscience, vol. 8, 2014.

[24] M. Seeber, R. Scherer, J. Wagner, T. Solis-Escalante, and G. R. MllerPutz, "High and low gamma eeg oscillations in central sensorimotor areas are conversely modulated during the human gait cycle," NeuroImage, vol. 112, pp. 318 - 326, 2015.

[25] Y. Zhang, G. Zhou, J. Jin, Q. Zhao, X. Wang, and A. Cichocki, "Sparse bayesian classification of eeg for brain-computer interface," IEEE transactions on neural networks and learning systems, vol. 27, no. 11 , pp. 2256-2267, 2016.

[26] Y. Zhang, Y. Wang, G. Zhou, J. Jin, B. Wang, X. Wang, and A. Cichocki, "Multi-kernel extreme learning machine for eeg classification in braincomputer interfaces," Expert Systems with Applications, vol. 96, pp. 302-310, 2018

[27] Y. Zhang, G. Zhou, J. Jin, X. Wang, and A. Cichocki, "Frequency recognition in ssvep-based bci using multiset canonical correlation analysis," International journal of neural systems, vol. 24, no. 04, p. 1450013, 2014

[28] C.-C. Chang and C.-J. Lin, "Libsvm: a library for support vector machines," ACM transactions on intelligent systems and technology (TIST), vol. 2, no. 3, p. 27, 2011.

[29] L. Breiman, "Random forests," Machine learning, vol. 45, no. 1, pp. 5-32, 2001.

[30] A. Karpathy and L. Fei-Fei, "Deep visual-semantic alignments for generating image descriptions," in Proceedings of the IEEE Conference on Computer Vision and Pattern Recognition, 2015, pp. 3128-3137.

[31] K. Gregor, I. Danihelka, A. Graves, D. J. Rezende, and D. Wierstra, "Draw: A recurrent neural network for image generation," arXiv preprint arXiv:1502.04623, 2015.

[32] D. Silver, A. Huang, C. J. Maddison, A. Guez, L. Sifre, G. Van Den Driessche, J. Schrittwieser, I. Antonoglou, V. Panneershelvam, M. Lanctot et al., "Mastering the game of go with deep neural networks and tree search," Nature, vol. 529, no. 7587, pp. 484-489, 2016.

[33] J. Li and A. Cichocki, "Deep learning of multifractal attributes from motor imagery induced eeg," in International Conference on Neural Information Processing. Springer, 2014, pp. 503-510.

[34] J. Li, Z. Struzik, L. Zhang, and A. Cichocki, "Feature learning from incomplete eeg with denoising autoencoder," Neurocomputing, vol. 165 , pp. 23-31, 2015.

[35] S. Jirayucharoensak, S. Pan-Ngum, and P. Israsena, "Eeg-based emotion recognition using deep learning network with principal component based covariate shift adaptation," The Scientific World Journal, vol. 2014, 2014.

[36] A. B. Said, A. Mohamed, T. Elfouly, K. Harras, and Z. J. Wang, "Multimodal deep learning approach for joint eeg-emg data compression and classification," in Wireless Communications and Networking Conference (WCNC), 2017 IEEE. IEEE, 2017, pp. 1-6.

[37] Y. LeCun, L. Bottou, Y. Bengio, and P. Haffner, "Gradient-based learning applied to document recognition," Proceedings of the IEEE, vol. 86, no. 11 , pp. 2278-2324, 1998 .

[38] H. Cecotti and A. Graser, "Convolutional neural networks for p300 detection with application to brain-computer interfaces," IEEE transactions on pattern analysis and machine intelligence, vol. 33, no. 3, pp. 433445, 2011.

[39] P. W. Mirowski, Y. LeCun, D. Madhavan, and R. Kuzniecky, "Comparing svm and convolutional networks for epileptic seizure prediction from intracranial eeg," in Machine Learning for Signal Processing, 2008. MLSP 2008. IEEE Workshop on. IEEE, 2008, pp. 244-249.

[40] P. Mirowski, D. Madhavan, Y. LeCun, and R. Kuzniecky, "Classification of patterns of eeg synchronization for seizure prediction," Clinical neurophysiology, vol. 120, no. 11, pp. 1927-1940, 2009.

[41] N. Lu, T. Li, X. Ren, and H. Miao, "A deep learning scheme for motor imagery classification based on restricted boltzmann machines," IEEE 
Transactions on Neural Systems and Rehabilitation Engineering, vol. 25, no. 6, pp. 566-576, 2017.

[42] S. M. Plis, D. R. Hjelm, R. Salakhutdinov, E. A. Allen, H. J. Bockholt, J. D. Long, H. J. Johnson, J. S. Paulsen, J. A. Turner, and V. D. Calhoun, "Deep learning for neuroimaging: a validation study," Frontiers in neuroscience, vol. 8, 2014.

[43] C. Zhang, P. Lim, A. Qin, and K. C. Tan, "Multiobjective deep belief networks ensemble for remaining useful life estimation in prognostics," IEEE transactions on neural networks and learning systems, vol. 28 , no. 10 , pp. 2306-2318, 2017.

[44] A. Graves, A.-r. Mohamed, and G. Hinton, "Speech recognition with deep recurrent neural networks," in Acoustics, speech and signal processing (icassp), 2013 ieee international conference on. IEEE, 2013, pp. 6645-6649.

[45] Y.-L. Hsu, Y.-T. Yang, J.-S. Wang, and C.-Y. Hsu, "Automatic sleep stage recurrent neural classifier using energy features of eeg signals," Neurocomputing, vol. 104, pp. 105-114, 2013.

[46] E. D. Übeyli, "Analysis of eeg signals by implementing eigenvector methods/recurrent neural networks," Digital Signal Processing, vol. 19, no. 1, pp. 134-143, 2009.

[47] P. Bashivan, I. Rish, M. Yeasin, and N. Codella, "Learning representations from eeg with deep recurrent-convolutional neural networks," arXiv preprint arXiv:1511.06448, 2015.

[48] P. Thodoroff, J. Pineau, and A. Lim, "Learning robust features using deep learning for automatic seizure detection," in Machine Learning for Healthcare Conference, 2016, pp. 178-190.

[49] H. Dong, A. Supratak, W. Pan, C. Wu, P. M. Matthews, and Y. Guo, "Mixed neural network approach for temporal sleep stage classification," IEEE Transactions on Neural Systems and Rehabilitation Engineering, vol. PP, no. 99, pp. 1-1, 2017.

[50] J. Chai, G. Chen, P. Thangavel, G. N. Dimitrakopoulos, I. Kakkos, Y. Sun, Z. Dai, H. Yu, N. Thakor, A. Bezerianos, and J. Li, "Identification of gait-related brain activity using electroencephalographic signals," in 2017 8th International IEEE/EMBS Conference on Neural Engineering (NER), May 2017, pp. 548-551.

[51] H. Yu, M. S. Cruz, G. Chen, S. Huang, C. Zhu, E. Chew, Y. S. Ng, and N. V. Thakor, "Mechanical design of a portable knee-ankle-foot robot," in 2013 IEEE International Conference on Robotics and Automation, May 2013, pp. 2183-2188.

[52] P. Aricò, G. Borghini, G. Di Flumeri, N. Sciaraffa, A. Colosimo, and F. Babiloni, "Passive bci in operational environments: Insights, recent advances, and future trends," IEEE Trans. Biomed. Eng, vol. 64, no. 7 , pp. 1431-1436, 2017

[53] P. Cherubino, M. Caratù, E. Modica, D. Rossi, A. Trettel, A. G Maglione, R. Della Casa, M. DallOlio, R. Quadretti, and F. Babiloni, "Assessing cerebral and emotional activity during the purchase of fruit and vegetable products in the supermarkets," in Neuroeconomic and Behavioral Aspects of Decision Making. Springer, 2017, pp. 293-307.

[54] J. Li, Y. Chen, F. Taya, J. Lim, K. Wong, Y. Sun, and A. Bezerianos, "A unified canonical correlation analysis-based framework for removing gradient artifact in concurrent eeg/fmri recording and motion artifact in walking recording from eeg signal," Medical \& Biological Engineering \& Computing, vol. 55, no. 9, pp. 1669-1681, 2017.

[55] J. Li, G. Chen, P. Thangavel, H. Yu, N. Thakor, A. Bezerianos, and Y. Sun, "A robotic knee exoskeleton for walking assistance and connectivity topology exploration in eeg signal," in 2016 6th IEEE International Conference on Biomedical Robotics and Biomechatronics (BioRob), June 2016, pp. 1068-1073.

[56] Z. Yang, K. E. Merrick, H. A. Abbass, and L. Jin, "Multi-task deep reinforcement learning for continuous action control." in IJCAI, 2017, pp. 3301-3307.

[57] A. L. Maas, A. Y. Hannun, and A. Y. Ng, "Rectifier nonlinearities improve neural network acoustic models," in Proc. ICML, vol. 30, no. 1, 2013.

[58] G. E. Hinton, N. Srivastava, A. Krizhevsky, I. Sutskever, and R. R. Salakhutdinov, "Improving neural networks by preventing co-adaptation of feature detectors," arXiv preprint arXiv:1207.0580, 2012.

[59] J. Shore and R. Johnson, "Axiomatic derivation of the principle of maximum entropy and the principle of minimum cross-entropy," IEEE Transactions on information theory, vol. 26, no. 1, pp. 26-37, 1980.

[60] D. Kingma and J. Ba, "Adam: A method for stochastic optimization," arXiv preprint arXiv:1412.6980, 2014

[61] M. Bentlemsan, E. T. Zemouri, D. Bouchaffra, B. Yahya-Zoubir, and K. Ferroudji, "Random forest and filter bank common spatial patterns for eeg-based motor imagery classification," in 2014 5th International Conference on Intelligent Systems, Modelling and Simulation, Jan 2014, pp. 235-238.
[62] A. Schwarz, R. Scherer, D. Steyrl, J. Faller, and G. R. Mller-Putz, "A co-adaptive sensory motor rhythms brain-computer interface based on common spatial patterns and random forest," in 2015 37th Annual International Conference of the IEEE Engineering in Medicine and Biology Society (EMBC), Aug 2015, pp. 1049-1052.

[63] D. Steyrl, R. Scherer, J. Faller, and G. R. Müller-Putz, "Random forests in non-invasive sensorimotor rhythm brain-computer interfaces: a practical and convenient non-linear classifier," Biomedical Engineering/Biomedizinische Technik, vol. 61, no. 1, pp. 77-86, 2016.

[64] M. Anastasiadou, M. Christodoulakis, E. Papathanasiou, S. Papacostas, and G. Mitsis, "Unsupervised detection and removal of muscle artifacts from scalp eeg recordings using canonical correlation analysis, wavelets and random forests," Clinical Neurophysiology, vol. 128, no. 9, pp. 1755-1769, 2017, cited By 0.

[65] W. Chen, Y. Wang, G. Cao, G. Chen, and Q. Gu, "A random forest model based classification scheme for neonatal amplitude-integrated eeg," BioMedical Engineering OnLine, vol. 13, no. 2, p. S4, Dec 2014.

[66] F. Akram, S. M. Han, and T.-S. Kim, "An efficient word typing p300bci system using a modified t 9 interface and random forest classifier," Computers in biology and medicine, vol. 56, pp. 30-36, 2015.

[67] C. Donos, M. Dümpelmann, and A. Schulze-Bonhage, "Early seizure detection algorithm based on intracranial eeg and random forest classification," International journal of neural systems, vol. 25, no. 05, p. 1550023,2015

[68] Y.-R. Lee and H.-N. Kim, "A data partitioning method for increasing ensemble diversity of an esvm-based p300 speller," Biomedical Signal Processing and Control, vol. 39, pp. 53 - 63, 2018.

[69] S. Siuly and Y. Li, "Improving the separability of motor imagery eeg signals using a cross correlation-based least square support vector machine for brain-computer interface," IEEE Transactions on Neural Systems and Rehabilitation Engineering, vol. 20, no. 4, pp. 526-538, 2012.

[70] Y. Liu, W. Zhou, Q. Yuan, and S. Chen, "Automatic seizure detection using wavelet transform and svm in long-term intracranial eeg," IEEE Transactions on Neural Systems and Rehabilitation Engineering, vol. 20, no. 6, pp. 749-755, Nov 2012.

[71] A. Presacco, R. Goodman, L. Forrester, and J. L. Contreras-Vidal, "Neural decoding of treadmill walking from noninvasive electroencephalographic signals," Journal of neurophysiology, vol. 106, no. 4, pp. 1875-1887, 2011.

[72] W. Yi, S. Qiu, H. Qi, L. Zhang, B. Wan, and D. Ming, "Eeg feature comparison and classification of simple and compound limb motor imagery," Journal of NeuroEngineering and Rehabilitation, vol. 10, no. 1, p. 106, Oct 2013. 